\title{
Billiards, scattering by rough obstacles and optimal mass transportation
}

\author{
Alexander Plakhov*
}

\begin{abstract}
This article presents a brief exposition of recent results of the author on billiard scattering by rough obstacles. We define the notion of a rough body and give a characterization of scattering by rough bodies. Then we define the resistance of a rough body; it can be interpreted as the aerodynamic resistance of the somersaulting body moving through a rarefied medium. We solve the problems of maximum and minimum resistance for rough bodies (more precisely, for bodies obtained by roughening a prescribed convex set) in arbitrary dimension. Surprisingly, these problems are reduced to special problems of optimal mass transportation on the sphere.
\end{abstract}

Mathematics subject classifications: 37D50, 49Q10

Key words and phrases: Billiards, problems of minimal and maximal resistance, scattering by obstacles, optimal mass transportation, rough surface, Newtonian aerodynamics, shape optimization, free molecular flow.

Running title: Scattering by rough obstacles

\section{Introduction}

This review article summarizes recent results obtained by the author on scattering of the billiard in the complement of a bounded domain, on a class of problems of minimal and maximal resistance in rarefied media, and on related problems of optimal mass transportation.

\footnotetext{
*University of Aveiro, Department of Mathematics, Aveiro 3810-193, Portugal email: plakhov@ua.pt
} 


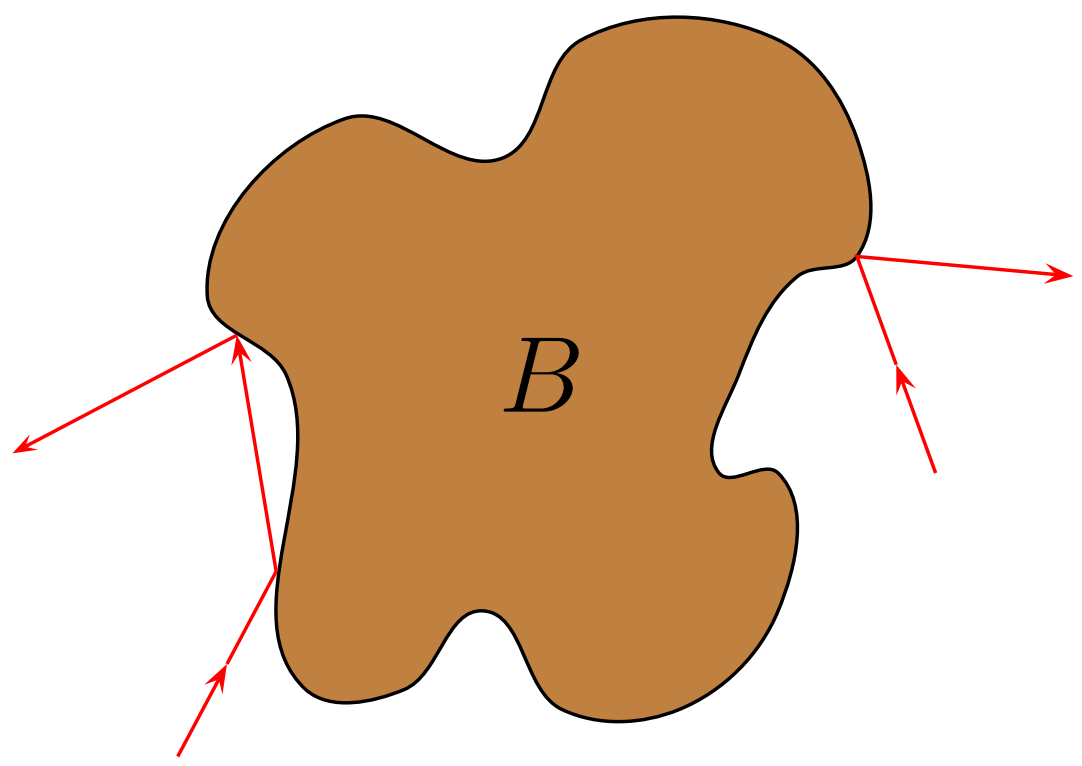

Figure 1: Billiard scattering in the complement of the domain $B$.

A bounded set $B$ with piecewise smooth boundary in Euclidean space $\mathbb{R}^{d}, d \geq 2$ will be called $a$ body. Note that a body is not necessarily connected.

The main object of our study is the billiard in $\mathbb{R}^{d} \backslash B$ (see Fig. 1). It represents a suitable model (in the three-dimensional case) for modeling interaction of a physical body with an incident flow, provided that the flow density is very low. A particle of the flow comes from infinity, makes one or several elastic reflections from the body surface, and goes to infinity again.

Two physical assumptions are adopted here.

1. The flow is so rare that the mutual interaction of particles can be neglected. In physical terms this means that the Knudsen number (the ratio of the particle mean free path to the body size) is large. Such flows are called in physics free-molecular flows.

2. All collisions of the flow particles with the body are perfectly elastic.

The conditions 1 and 2 can be satisfied, for example, in the case of artificial satellites moving on low Earth orbits, if the interaction of atmospheric particles with the satellite surface is specular (elastic). A possibility of fabricating satellites with specular surface was argued in [3]; nowadays, however, such an interaction is mostly diffuse [4]. Geometrical optics provides another physical field of applications for our theory.

The focus of this paper is on the notion of roughness. Note that rough surfaces have been intensively studied in natural sciences for more than 100 
years in various contexts. For instance, much work has been done on heat transfer through a rough interface between two media (see, e.g., [1]). A large part of contact mechanics is intended to describe the contact between two rough surfaces [6]. Much interest is attracted to diffraction of electromagnetic waves by randomly rough obstacles [5]. The roughness has been modeled in many different ways, depending on the specific phenomenon under study. Some researchers consider periodic functions with small period and amplitude, some others use so-called fractal functions. Another popular model is a random (Gaussian or non-Gaussian) function, with the correlation of function values depending on the values of the arguments.

On the contrary to these models, we are interested in characterizing all geometrically possible kinds of roughness. We will define the law of billiard scattering by a rough surface and characterize the class of all possible scattering laws.

We will also consider a class of problems of minimal and maximal resistance for moving bodies, which can be viewed as a long-going generalization of Newton's problem of minimal resistance. In these models, the body slowly and uniformly rotates (somersaults) in the course of translational motion. The problem consists in choosing a roughness on the surface of a convex body in such a way that the resulting rough body has minimal or maximal mean resistance. Surprisingly enough, these problems can be solved by reducing them to some special problems of optimal mass transportation, where the incident and reflected flows of particles are identified with the initial and final mass distributions, the law of scattering is identified with the plan of transportation, the momentum imparted to the body by an individual particle, with the cost of transportation, and the resistance, with the total cost.

In conclusion, one of the main results of the paper can be summarized as follows. The resistance can be both increased and decreased by roughening the body surface. It is much easier to increase the resistance than to decrease it. In the 2D case the greatest value of decrease is nearly $1 \%$, and of increase, $50 \%$ [8]. In the 3D case the greatest value of decrease is nearly $3 \%$, and of increase, $100 \%$ [9].

The paper is organized as follows. In section 2, the notions of scattering law and resistance of a (generally nonconvex) body are introduced and a motivating example explaining the meaning of resistance is given. In section 3 , we consider several examples of bodies obtained from a given convex set by a small (in $C$, but not in $C^{1}$ ) perturbation of its boundary, and calculate the rate of increase for the resistance of the perturbed bodies. Then we define a rough body and the law of scattering on it. In section 4, we formulate the characterization theorem for laws of scattering by rough bodies and outline its proof. Next we state the problems of maximal and minimal resistance 


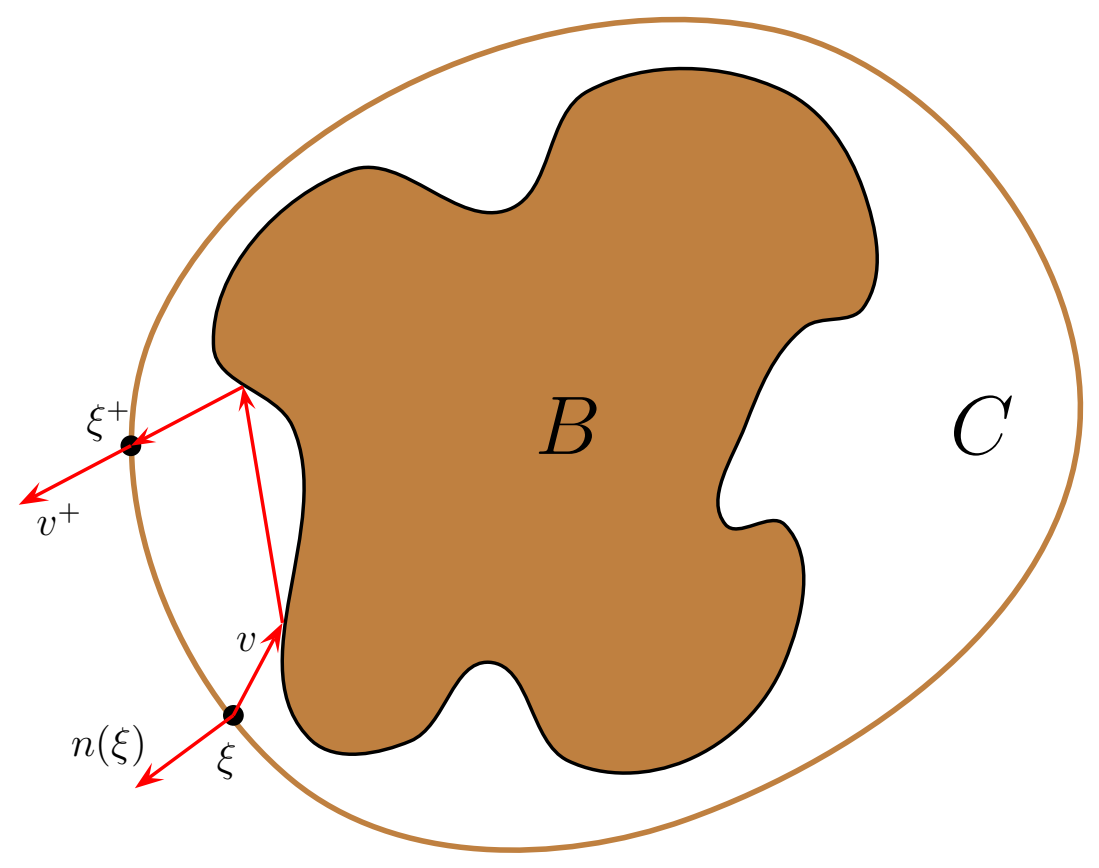

Figure 2: The law of scattering by the body $B$.

for rough bodies and solve them by reducing to special problems of optimal mass transportation on the sphere.

\section{Definition of scattering law and resistance}

In this section, we define the notion of the law of scattering by a (generally nonconvex) body $B$, and the (aerodynamic) resistance of the body. We also discuss a motivating example.

In what follows, $\partial C$ denotes the boundary of $C$, and $S^{d-1}$ denotes the $(d-1)$-dimensional unit sphere. Suppose that $X \subset \mathbb{R}^{n}$ and $Y \subset \mathbb{R}^{m}$ are Borel sets, $\mu$ is a Borel measure on $X$, and $T: X \rightarrow Y$ is a Borel map. Then the measure $T^{\#} \mu$ on $Y$ is defined by $T^{\#} \mu(A)=\mu\left(T^{-1}(A)\right)$ for any Borel $A \subset Y$ and is called push-forward of $\mu$ through $T$.

Take an (arbitrary) convex set $C$ containing $B$. For any particle incident on $B$ fix the velocity of incidence $v$, the final velocity $v^{+}$, and the outer normal to $\partial C$ at the point where the particle intersects $\partial C$ for the first time. Denote by $n(\xi)$ the unit outer normal to $\partial C$ at $\xi \in \partial C$ and denote by $\tau_{C}$ the surface measure of $C$. 
Define the measure $\mu_{C}$ on $\partial C \times S^{d-1}$ by

$$
d \mu_{C}(\xi, v)=(n(\xi) \cdot v)_{-} d \xi d v,
$$

where dot denotes the scalar product, $z_{-}:=\max \{0,-z\}$ and $z_{+}:=\max \{0, z\}$ denote the negative and positive parts of the real value $z$, and $d \xi$ and $d v$ are Hausdorff $(d-1)$-dimensional measures on $\partial C$ and $S^{d-1}$, respectively. Note that $\mu_{C}$ is a natural measure determining the number of incident particles per unit time.

For any particle with initial data $(\xi, v) \in \partial C \times S^{d-1}$ denote its final velocity by $v_{B, C}^{+}(\xi, v)$, and define the mapping $T=T_{B, C}: \partial C \times S^{d-1} \rightarrow$ $\left(S^{d-1}\right)^{3}$ by

$$
T:(\xi, v) \mapsto\left(v, v_{B, C}^{+}(\xi, v), n(\xi)\right)
$$

Definition 1. The push-forward measure $\nu_{B, C}:=T^{\#} \mu_{C}$ is called the law of billiard scattering by $B$.

Informally speaking, the measure $\nu_{B, C}$ defines the joint distribution of the triple $\left(v, v^{+}, n\right)$ for a randomly chosen incident particle. One can also imagine that we have million parallel flows falling on $B$, the corresponding million directions being uniformly distributed in $S^{d-1}$. For each particle of each flow (incident on $C$ ) determine the triple $\left(v, v^{+}, n\right)$. The collection of all these triples is distributed according to the measure $\nu_{B, C}$.

Remark 1. Note that two measures $\nu_{B, C_{1}}$ and $\nu_{B, C_{2}}$ corresponding to different ambient convex sets $C_{1}$ and $C_{2}$ are closely connected. Indeed, denote by $\pi_{v, v^{+}}:\left(S^{d-1}\right)^{3} \rightarrow\left(S^{d-1}\right)^{2}$ the projection $\pi_{v, v^{+}}\left(v, v^{+}, n\right)=\left(v, v^{+}\right)$; then the difference of the marginals $\pi_{v, v^{+}}^{\#} \nu_{B, C_{1}}-\pi_{v, v^{+}}^{\#} \nu_{B, C_{2}}$ is a (generally alternating) measure supported on the subspace $\left\{v=v^{+}\right\}$.

Definition 2. The resistance of the body $B$ is

$$
R(B)=\int_{\left(S^{d-1}\right)^{3}}\left(v-v^{+}\right) \cdot v d \nu_{B, C}\left(v, v^{+}, n\right) .
$$

Note that this definition does not depend on the choice of the ambient convex set $C$.

Remark 2. Let us explain the intuitive meaning of this definition. Consider a body $B$ that moves translationally in a rarefied medium and at the same time slowly and uniformly rotates around its center of masses. The force of resistance exerted by the medium on $B$ obviously depends on the time. We are considering the time average of the resistance along the direction 
of motion. The body rotation is so slow that it can be neglected when considering interaction of each individual particle with the body. Uniformity of rotation means the following. In the reference system connected with the body, the flow direction is represented by a unit vector $v(t)$ depending on time. Then for any continuous function $g$ on $S^{d-1}$ holds

$$
\lim _{T \rightarrow \infty} \frac{1}{T} \int_{0}^{T} g(v(t)) d t=\frac{1}{\left|S^{d-1}\right|} \int_{S^{d-1}} g(x) d x .
$$

An incident particle imparts to the body the momentum proportional to $v-v_{+}$(where the factor is the mass of the particle). The projection of the imparted momentum on the direction of the body's motion equals $\left(v-v^{+}\right) \cdot v$. Summing up over all particles incident on the body over a long period of time (and the summation amounts to integration over $\nu_{B, C}$ ), one gets $R(B)(1)$, that is, the mean value of the resistance force along the direction of the body's motion.

Remark 3. In the case where the body is convex, $B=C$, one easily calculates the corresponding scattering law and the resistance. Denote by $\tau_{C}$ the surface measure of $C$; then, using the law of elastic reflection $v^{+}=$ $v-2(v \cdot n) n$, one gets

$$
d \nu_{C, C}\left(v, v^{+}, n\right)=\left[(n \cdot v)_{-}\right] \delta\left(v^{+}-(v-2(v \cdot n) n)\right) d v d \tau_{C}(n)
$$

and

$$
R(C)=\int_{\left(S^{d-1}\right)^{2}} 2(v \cdot n)^{2}(v \cdot n)_{-} d v d \tau_{C}(n)=b_{d}|\partial C|,
$$

where $b_{d}=\int_{S^{d-1}} 2(v \cdot n)_{-}^{3} d v$. In particular, one has $b_{2}=8 / 3$ and $b_{3}=\pi$. Thus, we came to an important conclusion: the resistance of a convex body $R(C)$ is proportional to the area of its surface $|\partial C|$.

Let us give a motivating example. Consider a spaceship making a long galactic voyage. During the voyage it will traverse huge interstellar clouds, which will of course slow down its motion. The cloud temperature is zero, so the cloud particles stay at rest. The spaceship is inhabited, and constant movement of the crew results in a (very slow and chaotic) rotational motion of the ship.

Initially the spaceship is a convex body. The Mission Control Center wants to apply a riffling (roughening) on its surface so as to minimize the resistance when passing through the clouds, and thereby, to minimize the velocity loss. At the same time, a malicious member of the staff (presumably a spy from an extraterrestrial civilization) wants to maximize the ship resistance, and thus, prevent it from going too far. 


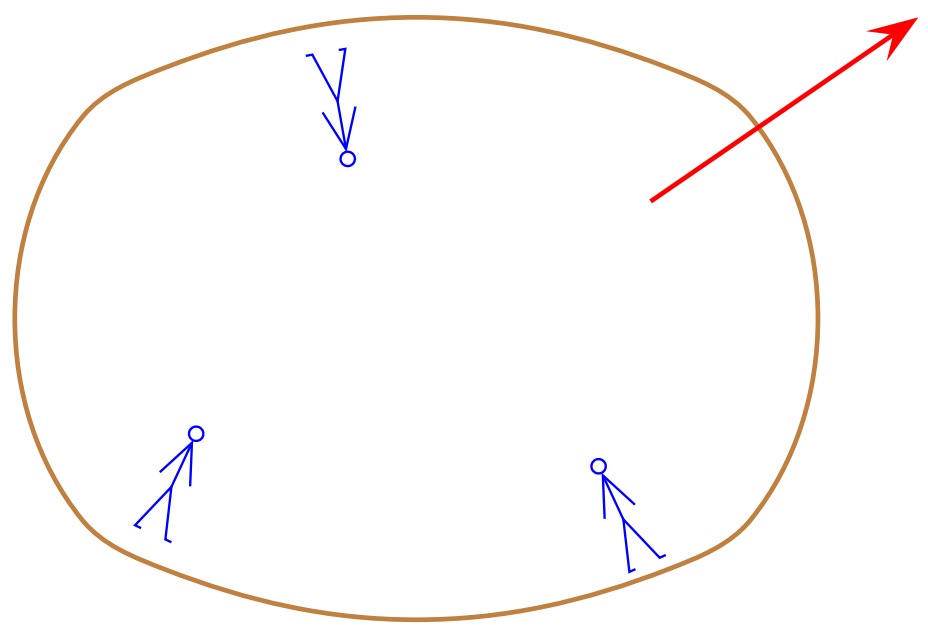

Figure 3: Motivating example: a spaceship.

Thus, the problem is twofold: minimize or maximize the resistance by riffling.

Remark 4. Note in passing that the problem of detecting the body of minimal resistance in the class of convex bodies of fixed volume can be easily solved. Indeed, according to the remark 3, the resistance of a convex body is proportional to its surface area, therefore the minimization problem amounts to the isoperimetric one: find the convex body of fixed volume and minimal surface area. The solution is a ball of given volume.

It is natural to wonder whether it is possible to further decrease the resistance of the ball by roughening its surface. The answer is by no means obvious; intuition tells us it is hardly possible. Surprisingly, the answer is positive, but the decrease value is very small: approximately $1.22 \%$ if $d=2$, $3.05 \%$ if $d=3$, and nearly $19 \%$ as $d \rightarrow \infty$.

Actually, we will state this question for an arbitrary convex body $C$. As we will see below, for any $C$ the resistance can be both decreased and increased by roughening its surface, and the decrease/increase ratios do not depend on $C$.

\section{Roughening the surface of a convex body}

Intuitively it seems to be much easier to make things worse than to make them better (increase rather than decrease the resistance), and this is really 


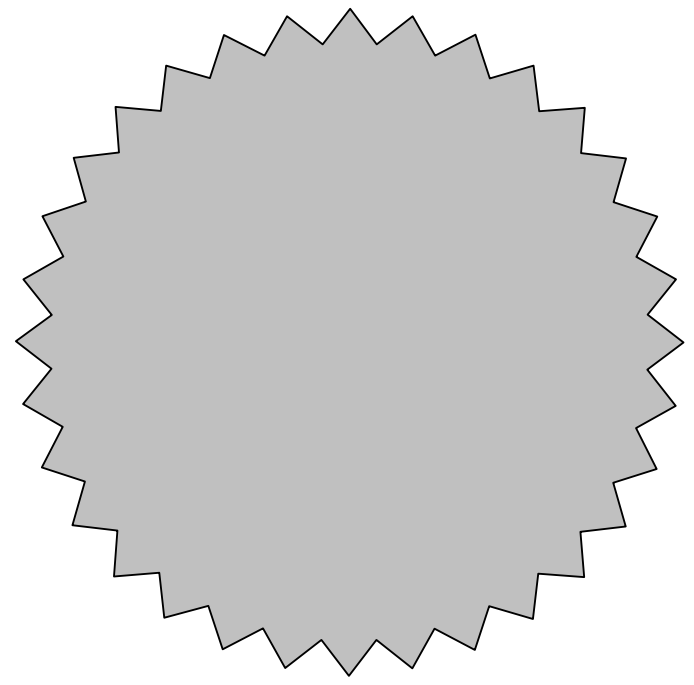

Figure 4: A body with triangular dimples on the boundary.

the case. Consider several examples of riffling in two dimensions.

Example 1. Take a circle $C$ with a suitable radius, so that its resistance equals 1. Substitute its boundary with an inscribed closed broken line with $2 m$ segments. The segments are of equal length; any two adjoint segments with the common point inside $C$ form the angle $45^{\circ}$ (see Fig. 4). Denote by $B_{m}$ the body bounded by this broken line, and by $C_{m}=\operatorname{Conv} B_{m}$, its convex hull. After a simple, but cumbersome calculation one determines the resistance, $R\left(B_{m}\right)=\sqrt{2}\left|\partial C_{m}\right| /|\partial C|$. Since the perimeter of a regular $2 m$ gon inscribed in a circumference approaches the length of the circumference as $m \rightarrow \infty, \lim _{m \rightarrow \infty}\left(\left|\partial C_{m}\right| /|\partial C|\right)=1$, one concludes that

$$
\lim _{m \rightarrow \infty} R\left(B_{m}\right)=\sqrt{2}
$$

As $m \rightarrow \infty$, the size of segments goes to 0 , so in the limit one gets a "rough body" with "infinitely small" dimples on its boundary, with the resistance $\sqrt{2}$ times greater than that of the original body (circle).

Example 2. Consider a more general case, where the angle at the "apex" of the triangular dimples equals $\alpha \in(0, \pi]$. The choice $\alpha=\pi / 2$ corresponds to the previous case, and if $\alpha=\pi$, one gets a regular $m$-gon. In fact, analytical formulas for all values of $\alpha$ can be derived, becoming more and more complicated as $\alpha \rightarrow 0$. In the case $\pi / 2 \leq \alpha \leq \pi$ the formula for the resistance of the corresponding $2 m$-gon $B_{m}^{\alpha}$ is still relatively simple. Each particle incident in a triangular dimple makes at most two reflections from 
the sides of the corresponding triangle. The calculation is made by taking into account, separately, 4 cases of reflection; namely, the 2 cases where the particle makes a single reflection from the left or from the right side, and the 2 cases where it makes a double reflection from the left and then from the right side or vice versa. After a cumbersome calculation one gets that the resistance equals $R\left(B_{m}^{\alpha}\right)=r(\alpha)\left|\partial C_{m}\right| /|\partial C|$, where

$$
\begin{aligned}
r(\alpha) & =\frac{3}{2}\left(1-\sin \frac{\alpha}{2}\right)+\frac{3}{4} \sin \frac{3 \alpha}{2}+\frac{1}{4} \sin \frac{5 \alpha}{2}-\frac{1}{2} \cos \alpha+ \\
& +\frac{3(1-\cos 2 \alpha)\left(1-\sin \frac{\alpha}{2}\right)}{4 \sin \frac{\alpha}{2}}+\frac{\cos 3 \alpha-9 \cos \alpha}{8 \sin \frac{\alpha}{2}} .
\end{aligned}
$$

That is, the resistance of the resulting "rough body" with infinitesimal dimples is $r(\alpha)$ times greater than that of the original body. In particular, one has

$$
r(\pi)=1, \quad r(2 \pi / 3)=\frac{5}{8}+\frac{1}{\sqrt{3}} \approx 1.2024 \quad \text { and } \quad r(\pi / 2)=\sqrt{2} \approx 1.4142 .
$$

Example 3. Consider the so-called Lorentz gas, that is, a collection of identical circles with centers at vertices of a rectangular lattice. The lattice size $\varepsilon$ is small, and the radius of the circles $\delta$ is even much smaller. The body $B_{\varepsilon, \delta}$ is the union of all the circles entirely contained in a prescribed convex body $C$; see Fig 5. Almost every particle, after getting into the body $B_{\varepsilon, \delta}$, eventually escapes; moreover, as $\varepsilon \rightarrow 0$ and $\delta / \varepsilon \rightarrow 0$, the mean distance between the points of "getting in" and "getting away" tends to 0 , and the velocities of getting in, $v$, and getting away, $v^{+}$, become independent. Using these properties, one easily calculates the limiting resistance, $\lim _{\varepsilon \rightarrow 0, \delta / \varepsilon \rightarrow 0} R\left(B_{\varepsilon, \delta}\right)=r$, where

$$
r=\frac{3}{4}\left(1+\frac{\pi^{2}}{16}\right) \approx 1.2126
$$

Example 4. Consider a set $B_{m}$ obtained by removing $m$ small semicircles from a fixed circle $C$ (recall that the resistance of the circle is assumed to be $R(C)=1)$. The radius of each semicircle equals $\sin (\pi / m)$. The resistance equals $R\left(B_{m}\right)=\sigma(\alpha)\left|\partial C_{m}\right| /|\partial C|$, where

$$
\sigma(\alpha)=\frac{3 \pi}{8} \approx 1.1781
$$

That is, the resistance of the resulting "rough body" is $\sigma(\alpha)$ times greater than that of the original body. 


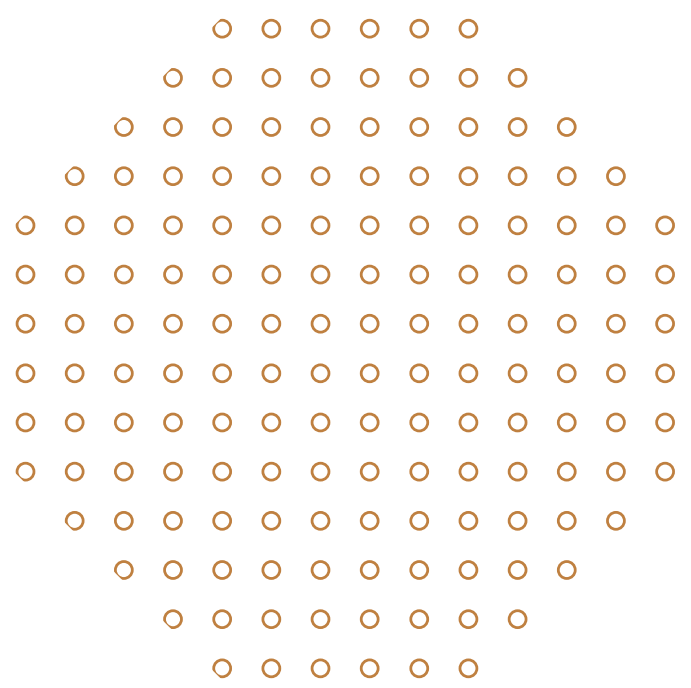

Figure 5: A body composed of small circles.

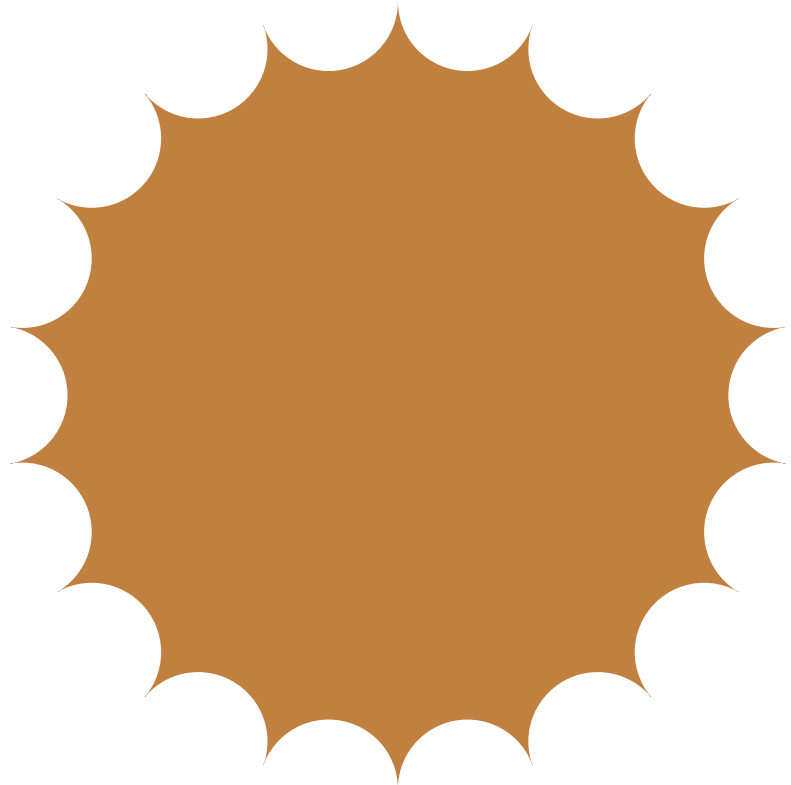

Figure 6: A body with semicircle-shaped dimples. 
These examples give us an idea how roughness should be defined. A rough body $\mathcal{B}$ is represented by a sequence of sets $B_{m}$ which are contained in $C$ and in the limit $m \rightarrow \infty$ fill it completely. Moreover, the measures defining the scattering laws by $B_{m}$ should converge as $m \rightarrow \infty$.

Definition 3. Let $C \subset \mathbb{R}^{d}$ be a bounded convex body. We say that a sequence of bodies $B_{m} \subset \mathbb{R}^{d}$ represents a rough body (or a body obtained by roughening $C$ ), if

(a) $B_{m} \subset C$ and $\lim _{m \rightarrow \infty}\left|C \backslash B_{m}\right|=0$;

(b) there exists the weak $\operatorname{limit}_{\lim } \lim _{m \rightarrow \infty} \nu_{B_{m}, C}=: \nu_{\mathcal{B}}$.

The limiting measure $\nu_{\mathcal{B}}$ is called the law of scattering by the rough body $\mathcal{B}$.

Two sequences $B_{m}$ and $B_{m}^{\prime}$ are called equivalent, if the corresponding limits coincide, $\lim _{m \rightarrow \infty} \nu_{B_{m}, C}=\lim _{m \rightarrow \infty} \nu_{B_{m}^{\prime}, C}$.

By definition, a rough body $\mathcal{B}$ is a class of equivalence of sequences of bodies satisfying (a) and (b).

\section{Characterization theorem and optimization of resistance}

Once the notion of rough body is defined, the following questions arise:

(i) Describe all possible laws of scattering by rough bodies.

(ii) Solve the problems of minimal and maximal resistance for rough bodies.

The characterization of the class of scattering laws is given by the following definition and theorem.

Denote by $\pi_{v, n}$ and $\pi_{v^{+}, n}$ the projections $\pi_{v, n}:\left(v, v^{+}, n\right) \mapsto(v, n)$ and $\pi_{v^{+}, n}:\left(v, v^{+}, n\right) \mapsto\left(v^{+}, n\right)$, and by $\pi_{d}$, the idempotent mapping $\pi_{d}:\left(v, v^{+}, n\right) \mapsto$ $\left(-v^{+},-v, n\right)$. Further, let $X \subset \mathbb{R}^{n}$ and $Y \subset \mathbb{R}^{m}$ be Borel sets equipped with Borel measures $\mu$ and $\nu$, respectively. Then the (Borel) product measure $\mu \otimes \nu$ on $X \times Y$ is uniquely defined by $\mu \otimes \nu(A \times B)=\mu(A) \cdot \nu(B)$ for any Borel $A \subset X$ and $B \subset Y$.

Definition 4. Denote by $\Gamma_{C}$ the set of measures $\nu$ on $\left(S^{d-1}\right)^{3}$ satisfying the following conditions: the marginals of $\nu$ are fixed $(\mathrm{a}, \mathrm{b})$ and $\nu$ is invariant with respect to the mapping $\left(v, v^{+}, n\right) \mapsto\left(-v^{+},-v, n\right)(\mathrm{c})$, that is,

(1) $\pi_{v, n}^{\#} \nu=(v \cdot n)_{-} u_{v} \otimes \tau_{C}$

(2) $\pi_{v^{+}, n}^{\#} \nu=\left(v^{+} \cdot n\right)_{+} u_{v^{+}} \otimes \tau_{C}$ 
(3) $\pi_{d}^{\#} \nu=\nu$,

where $u$ stands for the Lebesgue measure on $S^{d-1}$ and the corresponding subscripts $v$ and $v^{+}$indicate the coordinate which is used in the given case.

Theorem 1. The following holds true:

$$
\left\{\nu_{\mathcal{B}}: \mathcal{B} \text { is obtained by roughening } C\right\}=\Gamma_{C} .
$$

The proof of the theorem in the two-dimensional case is given in [8], and the full proof is given in [9].

Let us provide some arguments explaining why the theorem is true. First, one has to verify that each measure generated by a rough body, $\nu_{\mathcal{B}}$, satisfies the conditions (a), (b) and (c), and therefore belongs to $\Gamma_{C}$.

The condition (a) is easy to check. The marginal measure $\pi_{v, n}^{\#} \nu_{\mathcal{B}}$ describes the distribution of incident particles, according to $(v \cdot n)_{-} u_{v} \otimes \tau_{C}(n)$, and does not bear any information of scattering. The factor $\tau_{C}$ here stands for the distribution of $n$, and $(v \cdot n)_{-} u_{v}$, for the distribution of $v$ with fixed $n$. Here $(v \cdot n)_{-}$is the cosine of the angle of inclination of the incident particle with respect to the normal $n$; that is, the larger the angle, the smaller is the number of particles arriving at this angle.

The condition (b) describes the joint distribution of $\left(v^{+}, n\right)$, which is given by a formula almost identical to the case (a) (up to the change of the subscript sign from "-" to "+"). This formula is proved with the use of Liouville measure preservation property and of the fact that the mean distance between two points of intersection of $\partial C$ with a particle trajectory goes to zero as the number $m$ of the body $B_{m}$ in an approximating sequence goes to infinity. This fact is actually a variation of the mean free path formula for billiards (see, e.g., [2]). The condition (c) is a consequence of invariance of the billiard dynamics with respect to the time reversal $t \mapsto-t$ and of the fact mentioned above.

Second, one has to check that any measure $\nu \in \Gamma_{C}$ is a law of scattering by a rough body, $\nu=\nu_{\mathcal{B}}$. This part of the proof is much more difficult than the former one and is proved by making a direct construction. For this construction we refer to the papers [8] and [9].

Note that $\left(v-v^{+}\right) \cdot v=\frac{1}{2}\left|v-v^{+}\right|^{2}$.

Definition 5. The resistance of the rough body is the limit

$$
R(\mathcal{B}):=\lim _{n \rightarrow \infty} R\left(B_{n}\right)=\iiint_{\left(S^{d-1}\right)^{3}} \frac{1}{2}\left|v-v^{+}\right|^{2} d \nu_{\mathcal{B}}\left(v, v^{+}, n\right) .
$$

Now fix a convex body $C$ and consider all bodies $\mathcal{B}$ that can be obtained by roughening $C$. We study the following problems: find the rough bodies 
$\mathcal{B}_{\text {min }}$ and $\mathcal{B}_{\text {max }}$ that minimize and maximize the resistance $R(\mathcal{B})$. Multiplying the resistance by a positive constant $c$ and using Definition 5 , one comes to the equivalent formulation of the problems:

$$
\inf _{\mathcal{B}} c \iiint_{\left(S^{d-1}\right)^{3}} \frac{1}{2}\left|v-v^{+}\right|^{2} d \nu_{\mathcal{B}}\left(v, v^{+}, n\right) \text { and } \sup _{\mathcal{B}} c \iiint_{\left(S^{d-1}\right)^{3}} \frac{1}{2}\left|v-v^{+}\right|^{2} d \nu_{\mathcal{B}}\left(v, v^{+}, n\right) \text {. }
$$

Taking into account Theorem 1, the problems (2) take the form

$$
\left\{\begin{array}{l}
\inf \\
\sup
\end{array}\right\}_{\nu \in \Gamma_{C}} c \iiint_{\left(S^{d-1}\right)^{3}} \frac{1}{2}\left|v-v^{+}\right|^{2} d \nu\left(v, v^{+}, n\right) .
$$

It is convenient to choose the constant $c$ in such a way that $c R(C)=1$; with this choice, the values in (3) indicate the largest possible decrease and increase of resistance that can be achieved through roughening. One gets

$$
c=\left(\iiint_{\left(S^{d-1}\right)^{3}} \frac{1}{2}\left|v-v^{+}\right|^{2} d \nu_{C, C}\left(v, v^{+}, n\right)\right)^{-1}
$$

and thus, (3) reads as follows:

$$
\frac{\left\{\begin{array}{l}
\inf \\
\sup
\end{array}\right\}_{\nu \in \Gamma_{C}} \iiint_{\left(S^{d-1}\right)^{3}} \frac{1}{2}\left|v-v^{+}\right|^{2} d \nu\left(v, v^{+}, n\right)}{\iiint_{\left(S^{d-1}\right)^{3}} \frac{1}{2}\left|v-v^{+}\right|^{2} d \nu_{C, C}\left(v, v^{+}, n\right)} .
$$

Recall that the law of elastic reflection from the convex body $C$ is given by $d \nu_{C, C}\left(v, v^{+}, n\right)=\left[(n \cdot v)_{-}\right] \delta\left(v^{+}-(v-2(v \cdot n) n)\right) d v d \tau_{C}(n)$, and the corresponding integral defining the resistance of the convex body $C$ equals

$$
\iiint_{\left(S^{d-1}\right)^{3}} \frac{1}{2}\left|v-v^{+}\right|^{2} d \nu_{C, C}\left(v, v^{+}, n\right)=b_{d}|\partial C| .
$$

Note that instead of comparing total resistances of a rough and the convex bodies, it is more convenient to compare specific resistances of infinitely small pieces on their surfaces. In formulas this passage can be made by taking iterated integrals, first over $v$ and $v^{+}$, and then over $n$, that is,

$$
\frac{\left.\left\{\begin{array}{l}
\text { inf } \\
\sup
\end{array}\right\}_{\nu \in \Gamma_{C}} \int_{S^{d-1}} d \tau_{C}(n) \iint_{\left.S^{d-1}\right)^{2}} \frac{1}{2}\left|v-v^{+}\right|^{2} d \nu\right\rfloor_{n}\left(v, v^{+}\right)}{\left.\int_{S^{d-1}} d \tau_{C}(n) \iint_{\left(S^{d-1}\right)^{2}} \frac{1}{2}\left|v-v^{+}\right|^{2} d \nu_{C, C}\right\rfloor_{n}\left(v, v^{+}\right)},
$$


where $\nu\rfloor_{n}$ and $\left.\nu_{C, C}\right\rfloor_{n}$ are the corresponding conditional measures with $n$ fixed. Note that $\left.\nu_{C, C}\right\rfloor_{n}$ does not depend on the choice of $n$ and $\left.\nu_{0}:=\nu_{C, C}\right\rfloor_{n}$ satisfies $d \nu_{0}\left(v, v^{+}\right)=\left[(n \cdot v)_{-}\right] \delta\left(v^{+}-(v-2(v \cdot n) n)\right) d v$. Further, the measure $\nu\rfloor_{n}$ has fixed marginals $\lambda_{-}=(v \cdot n)_{-} u_{v}$ and $\lambda_{+}=\left(v^{+} \cdot n\right)_{+} u_{v^{+}}$.

The integrals $\left.\iint_{\left(S^{d-1}\right)^{2}} \frac{1}{2}\left|v-v^{+}\right|^{2} d \nu\right\rfloor_{n}\left(v, v^{+}\right)$and $\iint_{\left(S^{d-1}\right)^{2}} \frac{1}{2}\left|v-v^{+}\right|^{2} d \nu_{0}\left(v, v^{+}\right)$ in the numerator and denominator of (5) are interpreted as the specific resistances of the rough body and the convex body $C$, respectively, at the surface point with the outer normal $n$.

Definition 6. Denote by $\Gamma_{\lambda_{-}, \lambda_{+}}$the set of measures $\nu$ on $\left(S^{d-1}\right)^{2}$ with the marginals $\lambda_{-}$and $\lambda_{+}$, and define the functional $\mathcal{F}$ by

$$
\mathcal{F}(\nu)=\iint_{\left(S^{d-1}\right)^{2}} \frac{1}{2}\left|v-v^{+}\right|^{2} d \nu\left(v, v^{+}\right) .
$$

Taking into account this definition, the problem (5) can be written as follows:

$$
\frac{\left.\int_{S^{d-1}}\left\{\begin{array}{l}
\inf \\
\sup
\end{array}\right\}_{\nu\rfloor_{n} \in \Gamma_{\lambda_{-}, \lambda_{+}}} \mathcal{F}(\nu\rfloor_{n}\right) d \tau_{C}(n)}{\int_{S^{d-1}} \mathcal{F}\left(\nu_{0}\right) d \tau_{C}(n)} .
$$

Notice that the integrands in the numerator and the denominator in (7) are constant, therefore (7) reduces to the following problem in terms of specific resistances:

$$
\frac{\left\{\begin{array}{l}
\inf \\
\sup
\end{array}\right\}_{\nu \in \Gamma_{\lambda_{-}, \lambda_{+}}} \mathcal{F}(\nu)}{\mathcal{F}\left(\nu_{0}\right)} .
$$

In (8) we have the quotient of the resistance of an infinitesimal rough surface and the resistance of a flat infinitesimal surface of the same area. After finding the minimum and maximum measures, we verify that they are invariant with respect to the mapping $\left(v, v^{+}\right) \mapsto\left(-v^{+},-v\right)$.

The formulas (8) and (6) actually define some special problems of optimal mass transportation on the sphere, which are formulated as follows (see Fig. 7 for the case $d=3)$.

The sphere $S^{d-1}$ is divided into the lower hemisphere $S_{-}^{d-1}=S^{d-1} \cap$ $\{v: v \cdot n \leq 0\}$ and the upper hemisphere $S_{-}^{d-1}=S^{d-1} \cap\{v: v \cdot n \geq 0\}$. Both the measures, $\lambda_{-}$and $\lambda_{+}$, can be generated by vertical (parallel to $n$ ) translation of the Lebesgue measure concentrated on the equatorial disc $\{v:|v| \leq 1, v \cdot n=0\}$.

One is interested in transportation of the mass from the lower hemisphere (with the initial mass distribution $\lambda_{-}$) to the upper hemisphere (with the final 


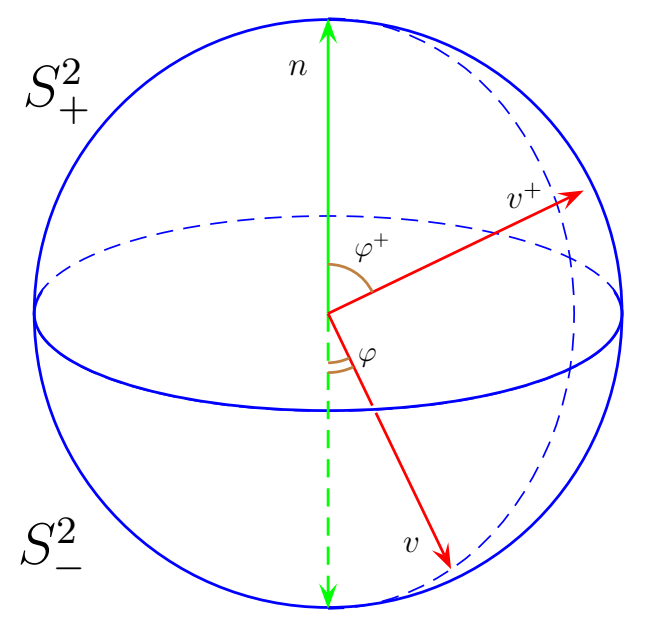

Figure 7: A mass transportation on the sphere.

mass distribution $\lambda_{+}$). A special roughness defines a special transportation plan. The local cost of transportation is the squared distance, $c\left(v, v^{+}\right)=$ $\frac{1}{2}\left|v-v^{+}\right|^{2}$. The total cost (specific resistance) is the integral $\mathcal{F}(\nu)(6)$. The denominator in $(8), \mathcal{F}\left(\nu_{0}\right)$, is the total cost of transportation induced by the symmetry with respect to the equatorial plane.

The transport plan providing the maximum cost is very easy to find; namely, it is generated by the central symmetry with respect to the origin. In other words, the optimal measure is supported on the subspace $v^{+}=-v$. The maximum resistance equals

$$
\frac{\sup _{\nu \in \Gamma_{C}} \mathcal{F}(\nu)}{\mathcal{F}\left(\nu_{0}\right)}=\frac{d+1}{2} .
$$

In particular, the maximum resistance equals 1.5 in the $2 \mathrm{D}$ case, and 2 in the $3 \mathrm{D}$ case.

Note that the optimal transportation problems (8), (6) are symmetric with respect to the rotation around the vertical axis $n$. By rearrangement arguments one can show that the transportation minimizing the cost is also rotationally symmetric and is performed along the meridians, and therefore, the corresponding problem reduces to a one-dimensional one. The reduced one-dimensional problem reads as follows:

$$
\text { find } \quad m_{d}=\inf _{\nu \in \Gamma_{\lambda_{d}, \lambda_{d}}} \iint_{[0, \pi / 2]^{2}} \frac{d^{2}-1}{4}\left(1+\cos \left(\varphi+\varphi^{+}\right)\right) d \nu\left(\varphi, \varphi^{+}\right) \text {; }
$$

here the measure $\lambda_{d}$ is given by $d \lambda_{d}(\varphi)=\sin ^{d-2} \varphi \cos \varphi d \varphi$, and $\Gamma_{\lambda_{d}, \lambda_{d}}$ is the set of measures on $[0, \pi / 2]^{2}$ with both marginals equal to $\lambda_{d}$. 


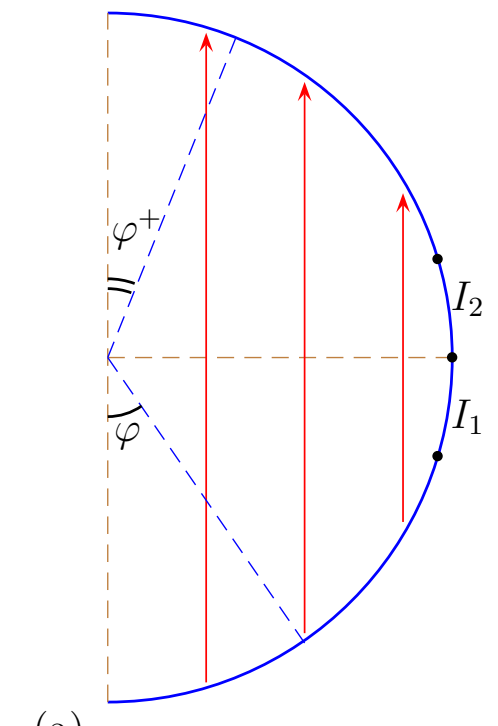

(a)

Figure 8: The transport from the lower to the upper quarter of the circle is indicated by arrows. The transport induced by reflection from the horizontal radius is shown in (a), and the optimal transport, in (b).

A simple argument shows that the measure $\nu_{0}$ given by $d \nu_{0}(\varphi)=\delta(\varphi-$ $\left.\varphi^{+}\right) \sin ^{d-2} \varphi \cos \varphi d \varphi$ is not a solution of (9) (and therefore, a flat surface does not minimize the resistance among all rough surfaces of the same area). Indeed, the problem (9) is visualized in Fig. 8 (a), where the transportation induced by $\nu_{0}$ is indicated by arrows. Since the cost function is the squared distance and the small intervals $I_{1}$ and $I_{2}$ in the right hand side of Fig. 8 (a) are "almost" rectilinear, the total transport cost can be decreased by reversing the monotonicity of the transportation from $I_{1}$ to $I_{2}$.

The optimal transportation can be described as follows (for a detailed exposition see $[7,9])$. The mass on both the lower and the upper quarter of the circle is divided into two parts, as shown in Fig. 8(b). The monotony of the transportation is different in these parts. Curiously enough, in the 2D case the mass of two mutually symmetric arcs on each circle quarter "splits along the curve". One part of the split mass is included in the monotone increasing transport plan, and the other part, in the monotone decreasing one. This splitting is schematically shown in Fig. 8(b), where arrows indicate the transport plan. The support of the optimal transportation in the $2 \mathrm{D}$ case is shown in Fig. 9. Here the splitting is represented by the rectilinear intervals $A_{1} \subset A$ and $B_{1}, B_{2} \subset B$ bounded by the dotted lines. The values 


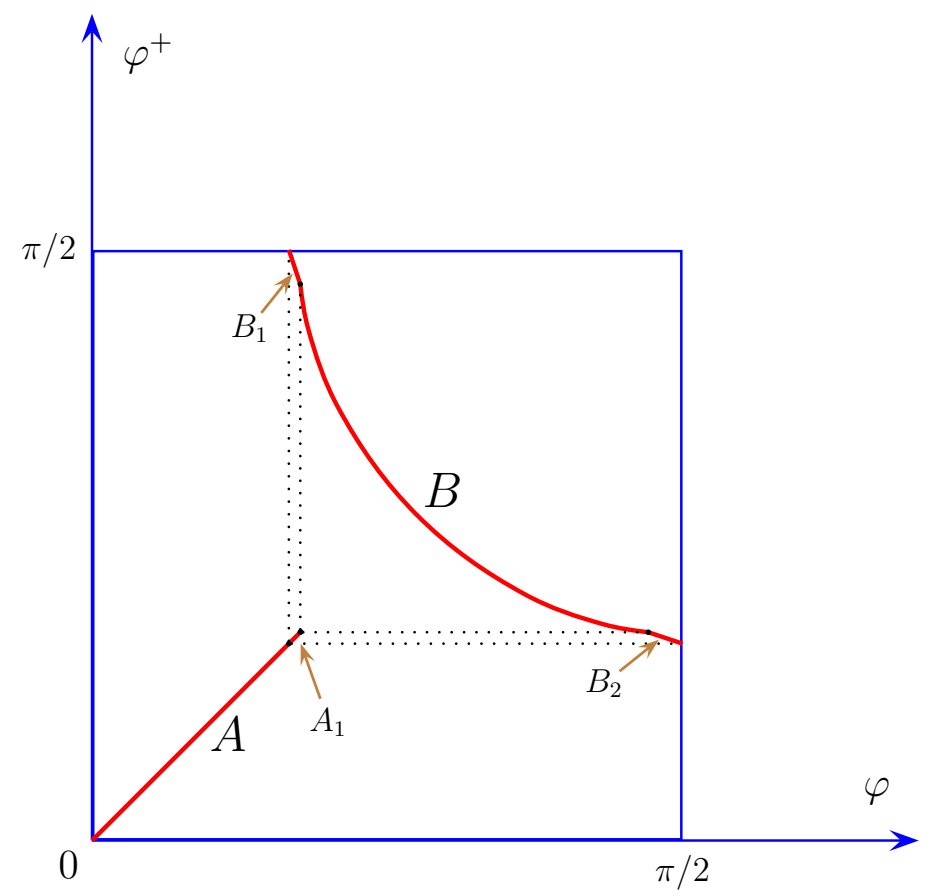

Figure 9: The support of the optimal measure in the 2D case is the union of two solid lines $A$ and $B$ inside the square.

of minimal resistance for $d$ from 2 to 11 are represented in Fig. 10. In particular, the minimal resistance equals $m_{2} \approx 0.98782$ in the $2 \mathrm{D}$ case, and $m_{3} \approx 0.96945$ in the $3 \mathrm{D}$ case. The limiting value of the minimal resistance equals $m_{\infty}=\lim _{d \rightarrow \infty} m_{d}=\int_{0}^{1} \sqrt{\ln z \ln (1-z)} d z \approx 0.791$ [9].

\section{Acknowledgements}

This work was partly supported by the Center for Research and Development in Mathematics and Applications (CIDMA) from the "Fundação para a Ciência e a Tecnologia" (FCT), cofinanced by the European Community Fund FEDER/POCTI, and by the FCT research project PTDC/MAT/113470/2009.

\section{References}

[1] G.A. Chechkin, A.L. Piatnitski and A.S. Shamaev. Homogenization. Methods and Applications Translations of Mathematical Monographs, 234. American Mathematical Society, Providence, RI (2007). 


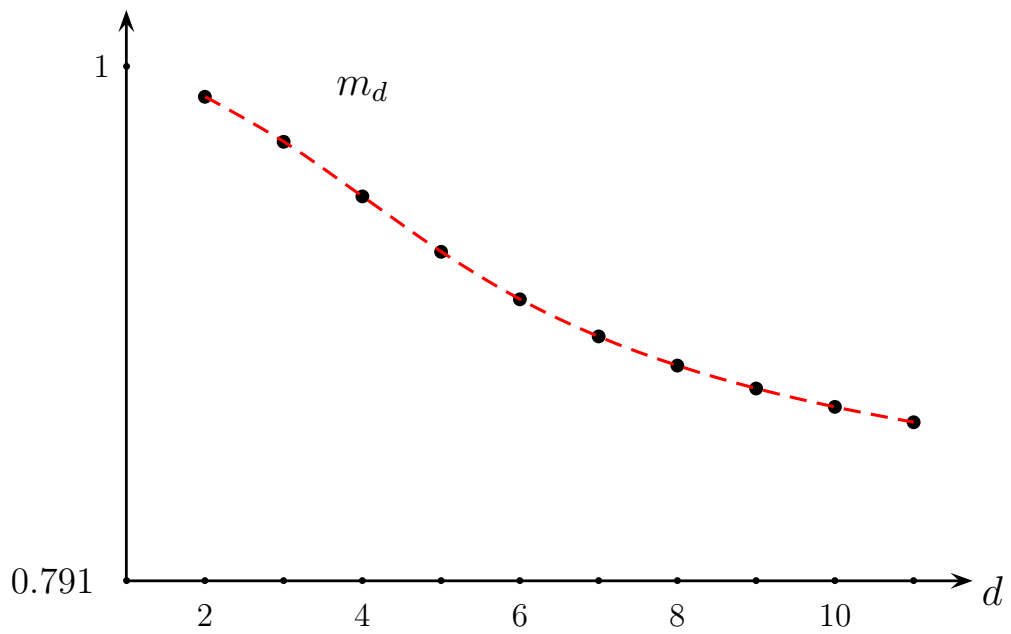

Figure 10: The minimal resistance $m_{d}$ for the values of dimension $d=$ $2,3, \ldots, 11$.

[2] N. Chernov. Entropy, Lyapunov exponents, and mean free path for billiards. J. Stat. Phys 88, 1-29 (1997).

[3] P. D. Fieseler. A method for solar sailing in a low Earth orbit. Acta Astronautica 43, 531-541 (1998).

[4] K. Moe and M. M. Moe. Gas-surface interactions and satellite drag coefficients. Planet. Space Sci. 53, 793-801 (2005).

[5] J.A. Ogilvy. Theory of wave scattering from random rough surfaces. Taylor \& Francis (1991).

[6] B.N.J. Persson. Contact mechanics for randomly rough surfaces. Surface Science Reports 61, 201227 (2006).

[7] A Plakhov. Newton's problem of the body of minimum mean resistance. Sbornik: Math. 195, 1017-1037 (2004).

[8] A. Plakhov. Billiard scattering on rough sets: Two-dimensional case. SIAM J. Math. Anal. 40, 2155-2178 (2009).

[9] A Plakhov. Scattering in billiards and problems of Newtonian aerodynamics. Russ. Math. Surv. 64, 873938 (2009). 